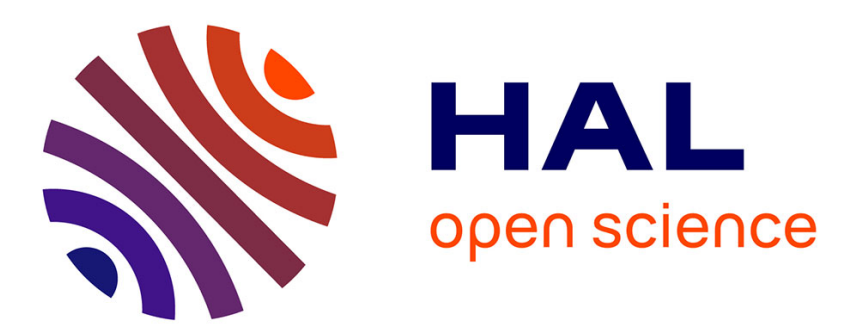

\title{
Experimental study of the influence of the degree of saturation on physical and mechanical properties in Tournemire shale (France)
}

\author{
Frédéric Valès, Duc Nguyen-Minh, Hakim Gharbi, Amel Rejeb
}

\section{- To cite this version:}

Frédéric Valès, Duc Nguyen-Minh, Hakim Gharbi, Amel Rejeb. Experimental study of the influence of the degree of saturation on physical and mechanical properties in Tournemire shale (France). Applied Clay Science, 2004, 26 (1-4), pp.197-207. 10.1016/j.clay.2003.12.032 . hal-00111413

\author{
HAL Id: hal-00111413 \\ https://hal.science/hal-00111413
}

Submitted on 1 Apr 2018

HAL is a multi-disciplinary open access archive for the deposit and dissemination of scientific research documents, whether they are published or not. The documents may come from teaching and research institutions in France or abroad, or from public or private research centers.
L'archive ouverte pluridisciplinaire $\mathbf{H A L}$, est destinée au dépôt et à la diffusion de documents scientifiques de niveau recherche, publiés ou non, émanant des établissements d'enseignement et de recherche français ou étrangers, des laboratoires publics ou privés. 


\title{
Experimental study of the influence of the degree of saturation on physical and mechanical properties in Tournemire shale (France)
}

\author{
F. Valès ${ }^{\mathrm{a}, *}$, D. Nguyen Minh ${ }^{\mathrm{a}}, \mathrm{H}$. Gharbi $^{\mathrm{a}}$, A. Rejeb ${ }^{\mathrm{b}}$ \\ ${ }^{\mathrm{a}}$ LMS Ecole Polytechnique, 91128 Palaiseau Cedex, France \\ ${ }^{\mathrm{b}}$ Institute of Radioprotection and Nuclear Safety, Fontenay aux Roses, France
}

\begin{abstract}
In addition to direct mechanical perturbations, an excavation influences rock conditions (mechanical, hydraulic and chemical properties). The internal fluids could be drastically modified and, in particular, the presence of water in the rock with regard to hydration and desiccation. The aim of the present study is to investigate the influence between the degree of saturation and the physical/mechanical properties for Tournemire shale rock. Anisotropy effects are also studied to identify hydromechanical behaviour. The different degrees of saturation are imposed by controlled suctions with continuous measurement of physical parameters such as weight and deformations. The volume variations (swelling or shrinkage behaviour) would result principally from the opening or closing of the inter-layers space. Uniaxial and triaxial compressive tests are carried out in order to attempt to establish a relationship between different mechanical parameters (elastic and failure data) and the sample saturation. The mechanical behaviour is sensitive to the saturation state of the shale with an important part of the stratification and its relative orientation to the mechanical stress.
\end{abstract}

Keywords: Shale; Hydromechanical behaviour; Imposed suction; Anisotropy; Saturation/de-saturation

\section{Introduction}

One of the major objectives of the hydromechanical behaviour studies of nuclear-waste storage in deep geological formations is to assess their stability and performance under variable hygrometry atmospheres. These perturbations can induce opening of new cracks in the disposal site, and loss of confinement properties of the geological barrier.

* Corresponding author. Tel.: +33-1-69-33-33-36; fax: +33-169-33-30-26.

E-mail address: vales@lms.polytechnique.fr (F. Valès).
In 1988, the Institute of Radioprotection and $\mathrm{Nu}-$ clear Safety (IRSN), a state agency in charge of safety assessment in the field of nuclear activities, selected the Tournemire site (Aveyron, France) to study the confining properties of argillaceous media. A 100-year-old railway tunnel gives access to a layer of Toarcian shales and marls. The tunnel is about $2 \mathrm{~km}$ long and $250 \mathrm{~m}$ deep; the shale layer in which the tunnel is dug out, is $250 \mathrm{~m}$ thick and lies between two limestone layers (Fig. 1) (Rejeb, 1999) (Cabrera Nunez et al., 2001).

In 1996, two lateral 30-m-long drifts were excavated from the central part of the tunnel. Different cracks could be observed in the drifts: (i) fractures at the wall of the gallery induced by decompression of the rock, 


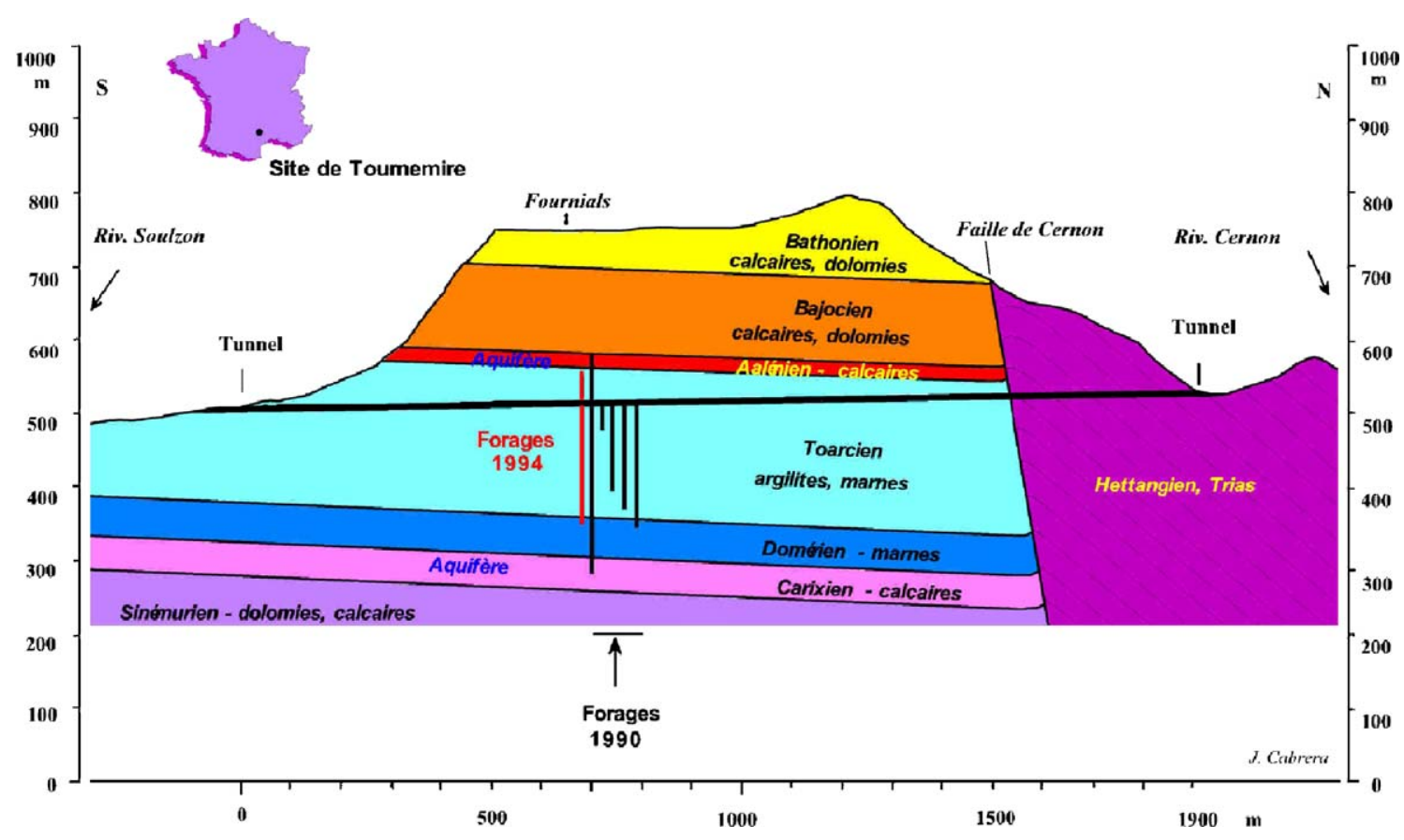

Fig. 1. Geological cross-section of the Tournemire site (Cabrera Nunez et al., 2001).

(ii) existing tectonic fractures in the rock mass, (iii) a set of regularly spaced $(20 \mathrm{~cm})$ horizontal cracks, developed shortly after excavation on the front faces, parallel to the bedding planes. Opening and closing of the cracks can be observed; they are governed by the de-saturation and re-saturation of the argillaceous medium due to the seasonal variations of temperature and humidity in the gallery. Cracks open in dry atmosphere (40\% RH, during winter) and close in water-saturated atmosphere (superior than $70 \% \mathrm{RH}$, during summer) in direct correlation with hydration/de-hydration of the rock. Daupley (1997) carried out an experimental characterization of shale behaviour under moisture and mechanical stress. In particular, the influence of the water potential variations on the shale behaviour was investigated (moisture transfer, relationship between the water content and the suction, and between the water content and the specific volume).

To understand the effects of the humidity conditions on shale rocks (Bergues and Nguyen Minh, 1999), the Laboratoire de Mécanique des Solides (LMS) conducted an experimental program to determine the effects of water saturation on the physical and mechanical properties of Tournemire shales. The correlation between the water in the material and the mechanical properties are required as input data to an hydromechanical (HM) model. Recently, Ramambasoa (2001) proposed a theoretical approach of the HM coupling based on use of the chemical potential as state variable describing the non-solid phases.

The aim of this work is to provide physical and mechanical data as a function of water saturation. To obtain different water saturations (from quasi-dry to quasi-saturated atmospheres), shale samples were put in equilibrium with atmospheres whose relative humidity was controlled. During these saturation or de-saturation steps, the changes in physical parameters (weight, length, local strains) are recorded. This measurement provides an evaluation of the kinetics of the mass transfer process. Furthermore, for the different saturation states, a mechanical characterization (compressive tests with and without confinement) is performed.

\section{General description of Tournemire shale}

The indurated argillaceaous rocks, or shales, of the Tournemire site are well-compacted rocks. The min- 


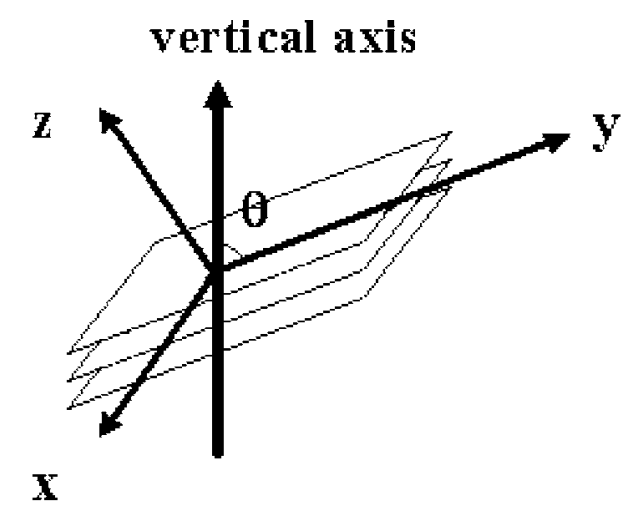

Fig. 2. Coordinate system associated to the shale bedding structure.

eralogy of this argillaceous rock (Cabrera Nunez et al., 2001) is characterized by a predominant clay fraction $(40-50 \%)$ made up of $15-20 \%$ of mixed layer illite/smectite, $15-25 \%$ of illite, $15-25 \%$ of kaolinite and smectite. Calcite is the predominant carbonate $(10-30 \%)$; dolomite is present in small proportions. Quartz occurs as grains $(10-20 \%)$. In the initial state (i.e. at the sample receipt at the laboratory), the degree of saturation is assumed to be close to $0.90-0.95$ with a native water content close to $3.5-4 \%$. The rock is not completely saturated, perhaps due to desiccation during the after sampling storage period. The total porosity is between $6.50 \%$ and $7.10 \%$ (Cabrera Nunez et al., 2001).

Tournemire shale shows thinly bedded clay minerals with a typical anisotropic texture (Rejeb, 1999; Cabrera Nunez et al., 2001). Subsequently, as illustrated schematically in Fig. 2, a coordinate system $(x, y, z)$ can be attached to the stratified structure of the shale (the $z$-axis is normal to the bedding planes which are defined by the two axes $x, y$ ). The angle $\theta$ is defined as the angle between the bedding plane $(x, y)$ and the vertical axis.

To investigate the effect of anisotropy, three sampling directions $\left(\theta=0^{\circ}, 45^{\circ}\right.$ and $\left.90^{\circ}\right)$ are used.

Tectonic fracturing and excavation-induced fractures are both present in the medium. The mechanical and hydrological environment could have important influence on the local composition and microstructure (newly formed porosity, fractures partially sealed by calcite, for example) (Charpentier et al., 2003). For this study, samples were bored in an area remote from the identified fractures to provide data of non-dis- turbed medium. In order to avoid changes in the water content, samples were bored with air, wrapped up with aluminium tight paper and paraffin, and stored at $5{ }^{\circ} \mathrm{C}$ before testing.

\section{Principle}

To obtain different degrees of water saturation, standard methods used in soil engineering were used during this testing program (Delage et al., 1998; Cuisinier and Masrouri, 2003; Al-Shayea, 2001). Rock samples are put in a tight box whose humidity is controlled; no mechanical loading is applied. The relative humidity imposes, using specific saline solutions, a suction pressure to the rock as given by Kelvin relationship:

$s=\rho_{\mathrm{w}} R T M^{-1} \ln (H / 100)$

where $s$ is the suction $(\mathrm{Pa}), \rho_{\mathrm{w}}$ the water density $(\mathrm{kg} /$ $\left.\mathrm{m}^{3}\right), R$ the universal gas constant $(\mathrm{J} / \mathrm{mol} / \mathrm{K}), T$ the absolute temperature $(\mathrm{K})$ and $H$ the relative humidity $(\% \mathrm{RH})$. Some examples of relative humidity and corresponding suction values are given in Table 1.

Different saline solutions were selected to cover the range of relative humidity measured over a year in the existing gallery.

During these suctions, different parameters are recorded continuously (see Fig. 3): weight, global deformations recorded with high-resolution Linear Variable Differential Transformer (LVDT) sensor, and local deformations measured with strain gauges (see Fig. 4).

When weight and strain evolutions are stabilized, the sample is assumed to be in equilibrium with the controlled humidity atmosphere. Then, mechanical

Table 1

Controlled suctions and saturations

\begin{tabular}{lllllll}
\hline $\begin{array}{l}\text { Relative } \\
\text { humidity } \\
\text { (\% RH) }\end{array}$ & $\begin{array}{l}\text { Initial } \\
\text { State }\end{array}$ & 98 & 76 & 50 & 36 & $<5$ \\
\hline $\begin{array}{l}\text { Suction } \\
(\mathrm{MPa})\end{array}$ & - & 2.8 & 38.4 & 97.0 & 142.9 & $>419$ \\
$w(\%)$ & 3.42 & 3.52 & 2.98 & 2.07 & 1.64 & 0.60 \\
$S$ & 0.90 & 0.95 & 0.85 & 0.60 & 0.45 & 0.20 \\
\hline
\end{tabular}




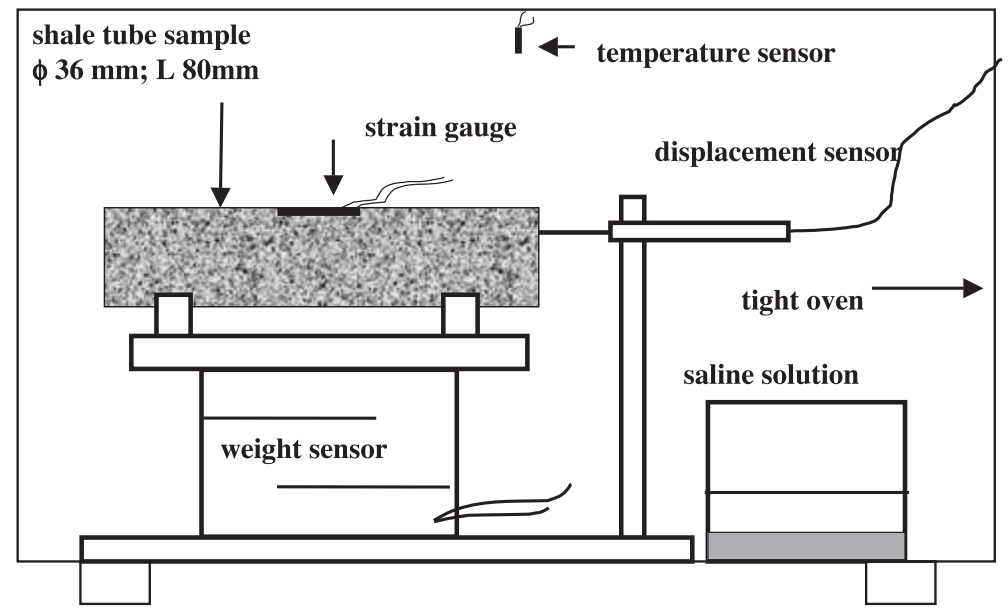

Fig. 3. Experimental saturation device.

tests are performed to obtain the requested parameters (elastic parameters and rock strength). Five suctions are conducted at different relative humidity steps (98\% RH, 76\% RH, 50\% RH, 36\% RH and close to $0 \% \mathrm{RH})$.

During the de-saturation/re-saturation tests and the mechanical characterization, deformations are important parameters; they allow to identify and quantify shrinkage or swelling, and to determinate the mechanical behaviour. One strain gauge is put in the axial direction of the sample (or axial stress direction) to measure longitudinal deformation $(L)$. Because of the transverse isotropic structure, two strain gauges are necessary to determine transverse defor-

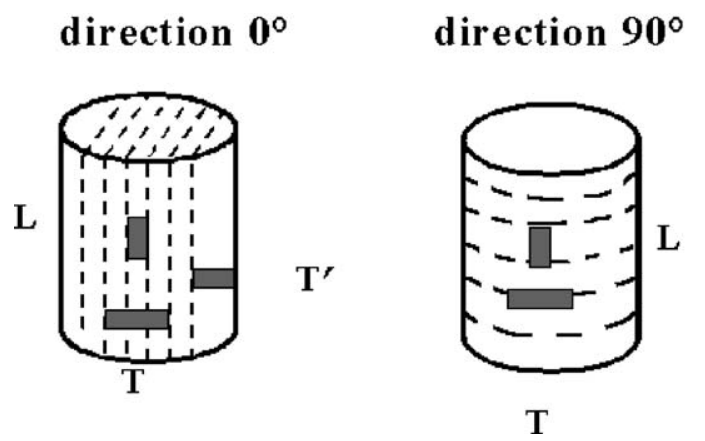

Fig. 4. Positions of the strain gauges (the dotted lines indicate the natural stratification; the gauges are mentioned by $\left.L, T, T^{\prime}\right)$. mations (in the planes perpendicular $(T)$ and parallel $\left(T^{\prime}\right)$ to the plane of isotropy) for the directions $\theta=0^{\circ}$ and $45^{\circ}$ (for $\theta=90^{\circ}$, the axial stress is normal the plane of isotropy so only one transverse strain gauge is necessary, whatever its location is). Fig. 4 illustrates the position of the gauges depending of the drilling direction.

For each sample, the water content $(w)$ and the degree of saturation $(S)$ are estimated. All the results of physical and mechanical measurements are presented as a function of the degree of saturation.

\section{Influence of saturation on physical properties}

De-saturations are conducted with increasing suction (from 2.8 to close to $400 \mathrm{MPa}$ ) - i.e. by decreasing the relative humidity from $98 \% \mathrm{RH}$ to close to $0 \%$ RH. Table 1 gives the water content $w$ and degree of saturation $S$ obtained after the different suctions. The three directions provide very similar results when weight loss or gain are concerned, so the values given in Table 1 are averaged without distinction with regard to sample orientation.

During each suction, the sample instrumentation allows continuous estimates of weight changes and deformation. The data give information on the correlation between the loss/gain of mass and shrinkage/ swelling. This method provides information on trans- 
a

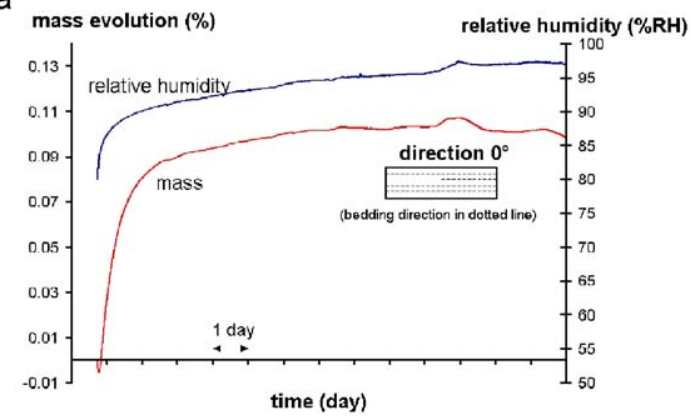

b

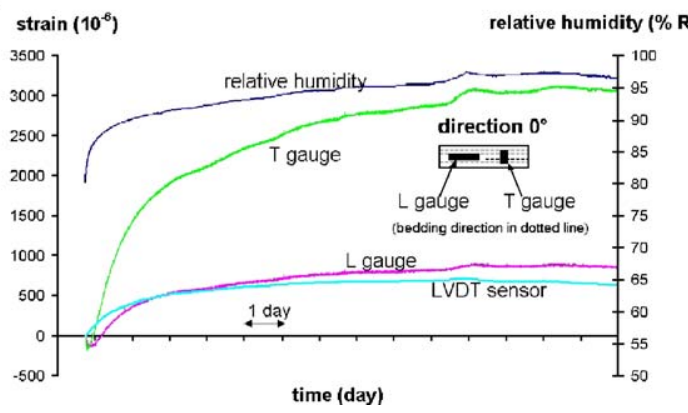

Fig. 5. Saturation at $98 \%$ RH: weight and strain evolutions versus time for a sample drilled at direction $\theta=0^{\circ}$. (a) Weight evolution during 98\% RH saturation. (b) Strains evolution during 98\% RH saturation.

fer kinetics, but the obtained data depend on the geometry of the sample.

Fig. 5 gives the evolution of weight and strains ( $L$ and $T$ ) for a sample drilled parallel to the bedding plane $\left(\theta=0^{\circ}\right.$ direction) from its initial state to $98 \%$ RH step. The evolution of the relative humidity is recorded; the increase in relative humidity at the beginning of the test is due to the opening of the tight box when samples are set. In Fig. 5a, the sample mass increases (by close to $0.1 \%$ ) over 5 days; this gain is in accordance with the estimation of $S$ at the initial state (close to 0.95); swelling is observed (see Fig. $5 b$ ): the gauge and the global displacement sensor in the bedding plane give identical strains (no scale effect) lower than the strain in the perpendicular plane (ratio 3).

This qualitative result suggests that the swelling could be due to opening of inter-layers. It can be inferred from these observations, that these inter-layer spaces may also represent privileged paths for moisture diffusion and water transfer.
Fig. 6 summarises the evolution of physical parameters for the other saturations with drier atmospheres.

- From weight and strain evolutions, the initial degree of saturation of the rock can be estimated close to $S=0.90$.

- The loss of weight is inversely proportional to the degree of saturation (refer to Fig. 6a). For the three directions, curves are parallel, with a shift due to an initial de-saturation of the rock samples

a

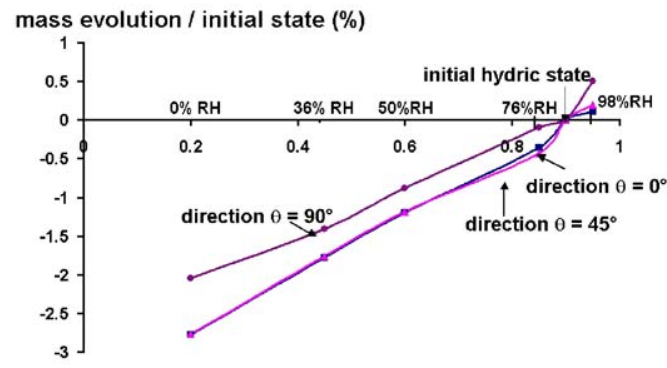

saturation $\mathrm{S}$

b

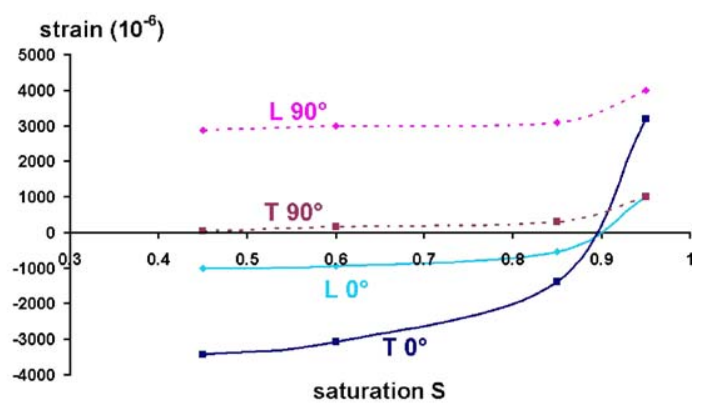

C

compressive wave velocity $(\mathrm{m} / \mathrm{s})$

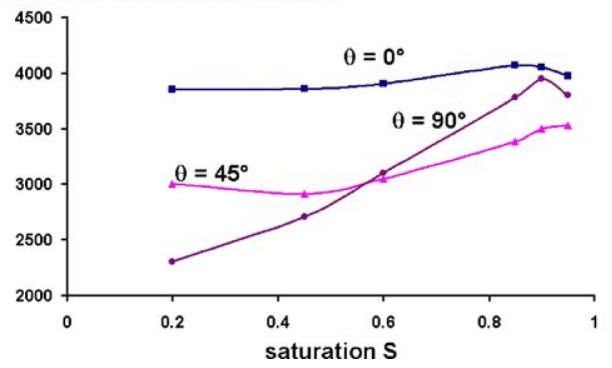

Fig. 6. Physical parameters evolutions versus the degree of saturation for all samples. (a) Weight evolution versus saturation. (b) Strains evolution versus saturation. (c) Compressive wave velocity evolution versus saturation. 
in the direction $\theta=90^{\circ}$. In the driest atmosphere (with silica gel at relative humidity close to $0 \%$ $\mathrm{RH}$ ), water is still present in the rock ( $S$ is close to 0.2 ). This suggests that two kinds of water are present in the rock: the first one, with low bonding energy, located in the "macro-connected" pores; and a second one adsorbed in the thin clayed layers or in small non-connected pores. Imposed vapour saturations act to regulate the low bonding water, but only drastic methods, like placing samples in an oven for the measurement of water content, can regulate the second type of water. This operation would damage the material with micro-cracking.

- Together with the weight loss or gain, Fig. 6b illustrates the evolution of strain gauge measurements (axial and transverse to the sample geometry) versus the degree of saturation after different suctions were imposed, for $\theta=0^{\circ}$ and $90^{\circ}$. The first saturation step, from native state to 98\% RH (corresponding to $S=0.95$ ), shows important inter-layer swelling aperture (for $\theta=0^{\circ}$ and $90^{\circ}$, gauges indicate inter-layer deformation values close to $4 \times 10^{-3}$ ). During the decrease of $S$ (from 0.95 to 0.45 ), for direction $\theta=0^{\circ}$, a significant shrinkage is observed in the direction perpendicular to the bedding (close to $6 \times 10^{-3}$ ); this shrinkage is less important in the bedding plane (close to $2 \times 10^{-3}$ ). However, for the de-saturation of the sample in the direction $\theta=90^{\circ}$, the shrinkage (in the layer plane and perpendicular) is small $\left(1 \times 10^{-3}\right)$ and quite insignificant for humidity below $76 \% \mathrm{RH}$. We cannot, at this time, provide a satisfactory explanation for this non-symmetric behaviour, but the constitutive heterogeneity of this rock could play important role in this phenomenon.

- For saturations lower than $76 \% \mathrm{RH}$, deformations are quite insignificant. The humidity of $76 \% \mathrm{RH}$ induces air penetration inside the pores with no deformation of the rock structure. For higher relative humidity, only water fills up the pores and the important global deformations are due to the deformation of the pores and a water transfer without air penetration.

- Another rock property was used: the velocity of compressive waves. This parameter is very sensitive to the texture of the material, particularly to the bedding orientation and to the presence of water or air in the pores. Fig. 6c shows the velocity of the compressive waves in the samples as a function of the degree of saturation. For the samples drilled parallel to the bedding, the velocity is quite constant (close to $4000 \mathrm{~m} / \mathrm{s}$ ), and does not depend on $S$. This result is consistent with what expected, as the wave is propagated through the dense layer and not through the inter-layers space, where, depending of $S$, the water is more-or-less present. For directions $\theta=45^{\circ}$ and $90^{\circ}$, during its travel through the sample, the wave crosses both the inter-layers space and water. We note in these two directions an increase of velocity with suction. Water increases sample density and makes wave propagation easier.

\section{Influence of saturation on mechanical properties}

Uniaxial and triaxial compressive tests with loading-unloading cycles are performed on cylindrical samples of diameter $36 \mathrm{~mm}$ and height $72 \mathrm{~mm}$. Strain gauges are put on the sample (see Fig. 4) according to the bedding position. The displacement rate is $0.2 \mathrm{~mm} / \mathrm{min}$. For the triaxial configuration, the confining pressure is $2.5 \mathrm{MPa}$. For compressive tests, samples are protected by a membrane to mitigate air exchanges which could modify the degree of saturation by water transfer. We assumed that the degree of saturation does not evolve during the test (test duration is close to $30 \mathrm{~min}$ ). The water content of each sample is measured when the mechanical test is over; it is quite similar to the estimation of $w$ made at the end of the imposed suction, providing some credit to the non-de-saturation hypothesis during the test.

Due to the limited number of samples, uniaxial compressive tests are conducted on three samples (the given values are averaged) and triaxial compressive tests on one sample.

The elastic behaviour of this rock can be qualified as transversely isotropic (as described by the generalized Hooke's law) (Rejeb, 1999; Cabrera Nunez et al., 2001; Niandou et al., 1997). The constitutive law of linear elastic, transversely isotropic medium, in the 
local coordinate system $(x, y, z)$ defined by Fig. 2, is as follows:

$$
\left[\begin{array}{c}
\varepsilon_{x} \\
\varepsilon_{y} \\
\varepsilon_{z} \\
\gamma_{y z} \\
\gamma_{z x} \\
\gamma_{x y}
\end{array}\right]=\left[\begin{array}{cccccc}
\frac{1}{E_{1}} & -\frac{v_{1}}{E_{1}} & -\frac{v_{2}}{E_{2}} & 0 & 0 & 0 \\
-\frac{v_{1}}{E_{1}} & \frac{1}{E_{1}} & -\frac{v_{2}}{E_{2}} & 0 & 0 & 0 \\
-\frac{v_{2}}{E_{2}} & -\frac{v_{2}}{E_{2}} & \frac{1}{E_{2}} & 0 & 0 & 0 \\
0 & 0 & 0 & \frac{1}{G_{2}} & 0 & 0 \\
0 & 0 & 0 & 0 & \frac{1}{G_{2}} & 0 \\
0 & 0 & 0 & 0 & 0 & \frac{2\left(1+v_{1}\right)}{E_{1}}
\end{array}\right]\left[\begin{array}{c}
\sigma_{x} \\
\sigma_{y} \\
\sigma_{z} \\
\tau_{y z} \\
\tau_{z x}
\end{array}\right]
$$

Five independent elastic parameters have to be identified: the Young moduli $E_{1}, E_{2}$ (respectively in the direction of the isotropic plane $(x, y)$ for $E_{1}$; and in $z$ direction, normal to the isotropic plane, for $E_{2}$ ); the Poisson's ratios $v_{1}, v_{2}\left(v_{1}\right.$ characterizes the transverse strain expansion in the plane of isotropy due to compressive stress in this plane; $v_{2}$ characterizes the same strain expansion in this plane of anisotropy due to compressive stress in the direction normal to it); the shear modulus $G_{2}$ for the plane normal to the plane of isotropy. The method (strain gauges location and relations of coordinate transformation) used to determinate the whole elastic coefficients is described in details in Niandou et al. (1997).

The Young moduli $E_{1}$ and $E_{2}$ are estimated in the first linear part of the $\sigma-\varepsilon$ curve during the monotonous loading. The shear modulus $G_{2}$ is deduced from the test performed in the out-of-axis orientation $\theta=45^{\circ}$. The cohesion $C$ and the friction angle $\phi$ are deduced from the representation of the two tests (performed at confining pressures equal to 0 and 2.5 $\mathrm{MPa}$ ) in Mohr plane.

For the initial water content (close to the natural condition), the determination of the mechanical parameters from the triaxial tests gives the following values: $E_{1}=20,550 \mathrm{MPa}, E_{2}=4500 \mathrm{MPa}, G_{2}=6500$ $\mathrm{MPa}, v_{1}=0.40$ and $v_{2}=0.23$ (these values are consistent with earlier results; Rejeb, 1999; Cabrera Nunez et al., 2001; Niandou et al., 1997). For all degrees of saturation, the Young moduli values during triaxial tests are higher than during uniaxial tests; the confinement stiffens the rock sample.
Fig. 7 illustrates the evolutions of mechanical parameters (elastic coefficients; triaxial compressive strength, $R_{\mathrm{tr}}$; cohesion, $C$; friction angle, $\phi$ ) as a function of the degree of saturation $S$ for the triaxial tests. Some general trends appear and suggest the following comments.

- For modulus $E_{1}$, values are quite constant (close to $20,000 \mathrm{MPa}$ ) for the range of saturation between 0.95 and 0.5 ; for lower values of saturation, modulus $E_{1}$ increases with suction. For the other elastic moduli $E_{2}, E_{45^{\circ}}$ and $G_{2}$, there is a quite linear correlation between the modulus and the degree of saturation; the modulus is divided by two when the saturation decreases. The Poisson's ratios decrease with suction.

- For all directions, there is an important decrease in triaxial compressive strength with the degree of saturation (refer to Fig. 7b). (This trend should be confirmed by other triaxial tests, but is similar for the results obtained for the uniaxial tests.) Values for compressive strength are close to 20-40 MPa for saturated samples and reach 70-90 MPa for the driest samples.

- The axial strain when compressive strength is reached, is not sensitive to the degree of saturation in the case of the $\theta=0^{\circ}$ direction, and in the case of other directions, there is a significant increase in maximum axial strain when increases the degree of saturation.

- The cohesion and friction angle (Fig. 7c and d) seem to be not correlated to the degree of saturation. For the samples drilled in the bedding plane direction, or $\theta=0^{\circ}$, the properties do not depend on the presence of water. For the other two directions $\left(\theta=45^{\circ}\right.$ and $\left.90^{\circ}\right)$, the cohesion and friction angle increase when drier states are considered.

The testing curves are given in Fig. 8 for the triaxial configuration in the case of the $\theta=0^{\circ}$ and $90^{\circ}$ directions. Anisotropy has the important following influences on mechanical behaviour:

- When the stress direction is parallel to the bedding, or $\theta=0^{\circ}$, (see Fig. 8a), for all suctions (except for the highest suction), strength and axial strain are quite constant (respectively, average values are 


\section{a}

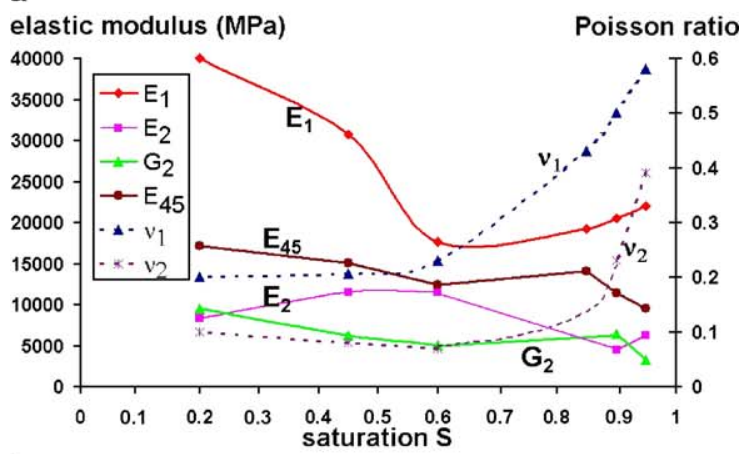

b

triaxial compressive strength $\mathrm{R}_{\mathrm{tr}}(\mathrm{MPa})$

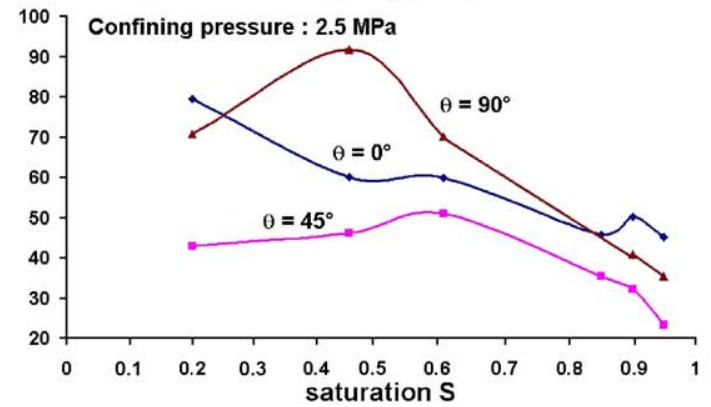

C

cohesion C (MPa)

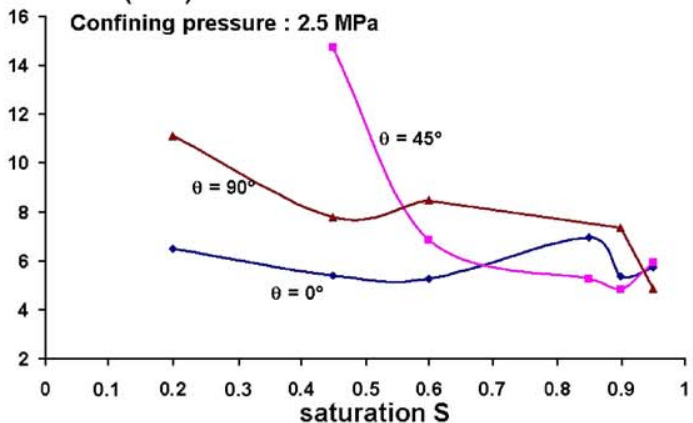

d

friction angle $\phi\left({ }^{\circ}\right)$

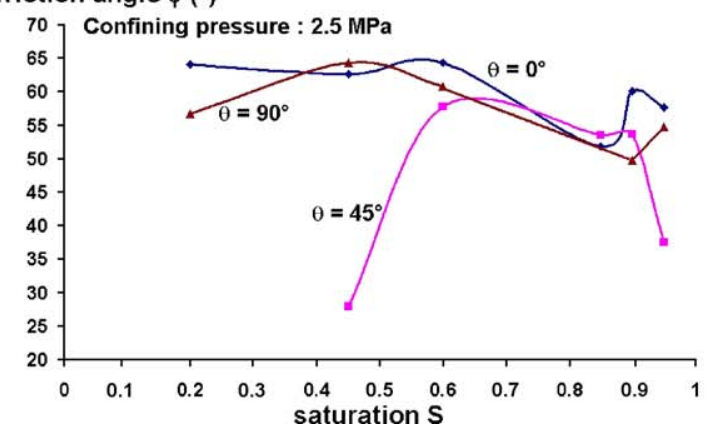

a

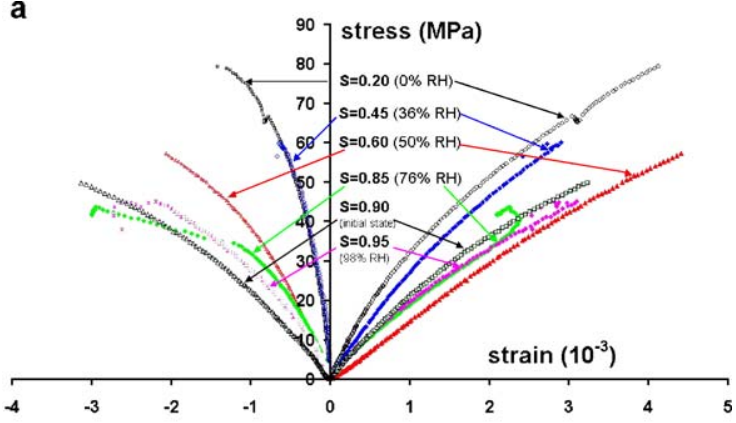

b

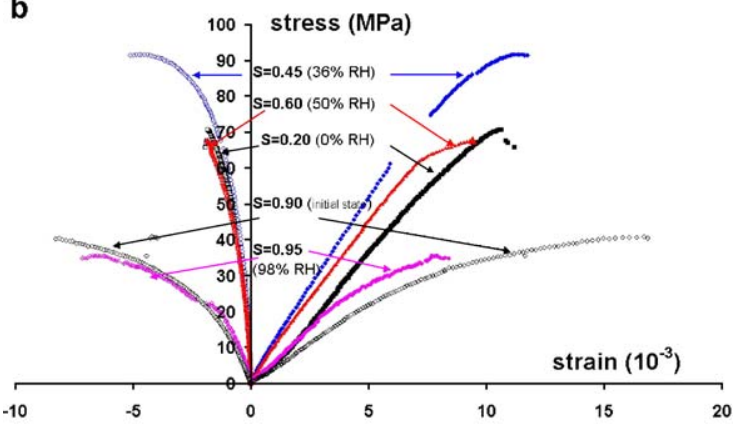

C

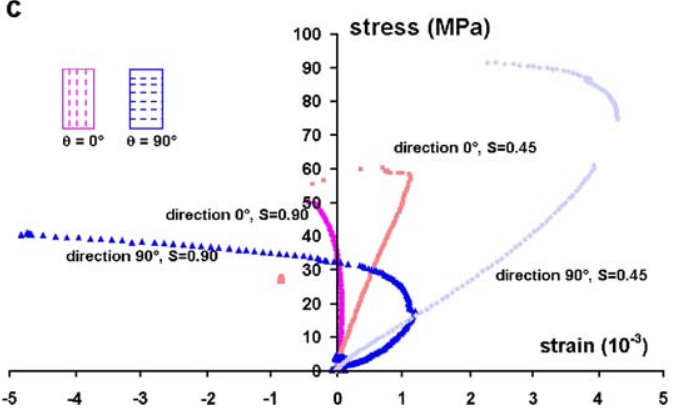

Fig. 8. Testing stress - strain curves (triaxial test with $2.5 \mathrm{MPa}$ confining). (a) Direction $\theta=0^{\circ}$. (b) Direction $\theta=90^{\circ}$. (c) Volume deformations for two degrees of saturation for samples drilled in perpendicular directions $\left(\theta=0^{\circ}\right.$ and $\left.90^{\circ}\right)$.

close to $50 \mathrm{MPa}$ for strength, and $3 \times 10^{-3}$ for axial strain). Only strains from transversal $(T)$ gauges are dependent on $S$ (the presence of water should allows the inter-layers movement like sliding).

Fig. 7. Evolution of mechanical parameters versus the degree of saturation. (a) Elastic parameters versus saturation. (b) Triaxial compressive strength versus saturation. (c) Cohesion versus saturation. (d) Friction angle versus saturation. 


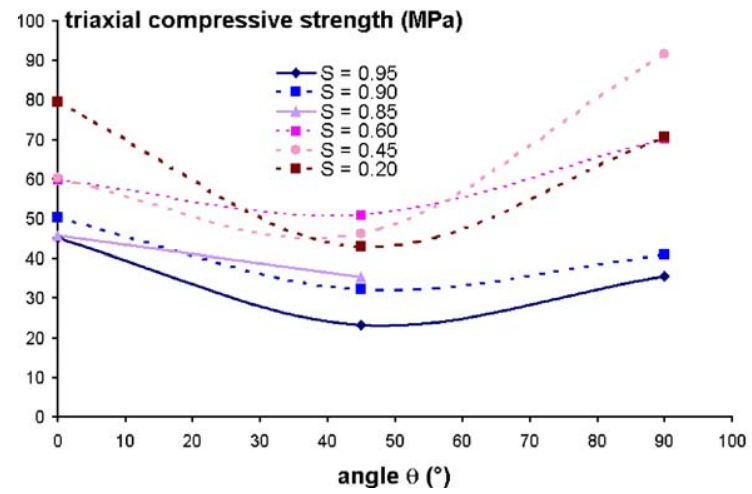

Fig. 9. Variation of triaxial compressive strength versus loading orientation $\theta$.

- When the stress direction is perpendicular to the bedding, or $\theta=90^{\circ}$, (see Fig. 8b), strains are larger than they were in the precedent configuration (longitudinal $(L)$ strains larger than $10^{-2}$ ). Indeed, a certain ductility is observed when saturation is above 0.80 , with low strength $(30-$ $40 \mathrm{MPa}$ ) and with important longitudinal $(L)$ (close to $1.5 \times 10^{-2}$ ) and transversal $(T)$ strains; for drier states, brittle behaviour becomes predominant with higher strength values and lower deformations (especially for transverse $(T)$ gauges), the sample stiffens. The important longitudinal deformation for the $\theta=90^{\circ}$ and $45^{\circ}$ orientations are in accordance with the observed shear fractures pattern (see Fig. 10).

- The volumetric deformations during mechanical loading are sensitive to the degree of saturation and to the bedding direction. Fig. 8c shows volumetric deformations for the $\theta=0^{\circ}$ and $90^{\circ}$ directions to the bedding, for two degrees of saturation $(S=0.90$ and $S=0.45)$. Volumetric deformations are a linear function of stress when low degrees of saturation are considered; the dilatancy threshold is high. For quasi-saturated state, volumetric deformations are a nonlinear function of dilatancy (especially for direction $\theta=90^{\circ}$ ). This increase of volume is more important when the deviatoric stress is applied perpendicularly to the bedding $\left(\theta=90^{\circ}\right.$ direction) and in presence of water. The dilatancy could originate in modifications of the rock organization through microfracturing and clayed grains or layer sliding in the presence of water. This dilatancy property is in accordance with the evolution of elastic moduli.

The as-measured triaxial compressive strength can also be presented as a function of the loading orientation $\theta$ for the different degrees of saturation (see Fig. 9). This representation allows to classify the anisotropy based on the curve shape as described by Ramamurthy (1993); for our case, the three values of $\theta$ give good trends. For a given degree of saturation, the failure stress depends strongly on the loading orientation. For every saturation, the curves present similar shape with two maximum values for $\theta=0^{\circ}$ and $90^{\circ}$, and a minimum value around $\theta=45^{\circ}$. More precisely, the degree of saturation seems to influence the relative position of the two maximum values. For high degrees of saturation $(S>0.85)$, the $\theta=0^{\circ}$ strength is higher than for the $\theta=90^{\circ}$ strength; the curve shape belongs to the shoulder type. For drier states $(S<0.60)$ (except for the driest value), this curve would be close to the $U$ type.

An analysis of fractures observed on tested samples has been carried out for the triaxial tests. Fig. 10 gives a schematic representation of the main failure modes in function of loading orientation and degree of saturation.

High S
$\theta=45^{\circ}$

Fig. 10. Schematization of main failure mode for two different degrees of saturation (the bedding planes are in dotted line and the failure is drawn in continuous line). 
The fracture angle is strongly depending on the loading orientation. With less influence, the presence of water has effects on the apparition of secondary cracks and on the angle between the fracture and the direction of the axial stress. As described in Niandou et al. (1997), two different failure modes occur in extension and shearing with a high dependency on the loading orientation.

For $\theta=0^{\circ}$ and $45^{\circ}$ loading orientations, failure occurs in the bedding plane with two different modes. For loading orientation in the bedding plane $\left(\theta=0^{\circ}\right)$, the failure is extensive, with several parallel cracks when water is present. For the $\theta=45^{\circ}$ loading orientation, the failure angle is close to $\theta$; failure is related to sliding of the bedding planes. For these two configurations, the presence of water allows the development of several cracks parallel to the main fracture. For $\theta=90^{\circ}$, the failure is mainly due to shear bands in the shale matrix. The angle between these bands and the bedding planes increases with suction.

\section{Conclusions}

The Tournemire shale with its bedding texture exhibits the characteristic behaviour of anisotropic rocks. Due to its $40 \%$ clay content, the properties of this shale is also dependent on moisture environment, which can be intrinsically characterized by its degree of saturation.

In this work, it is shown that these bedding planes are principally subject to moisture and water action when humidity environment changes. Thus these bedding planes seem to play a major role in the physical and mechanical properties of the rock.

Indeed, the volume variations, subsequent to gain or loss of water, would mainly result from opening or closing of the inter-layers space.

For the same reason, the velocities of compressive waves are sensitive to the presence of water, and to the wave direction; velocity increases with suctions except for direction parallel to the stratification.

The mechanical behaviour of the rock is also sensitive to its saturation state. In drier atmosphere, the elastic moduli, the compressive strengths and the cohesion are higher, while the Poisson's ratio is lower. With higher suction, the mechanical behaviour becomes brittle, especially for the direction perpen- dicular to the bedding. Anisotropy significantly influences the properties and the water dependence. Here again, it can be inferred that most mechanical changes occur along the inter-layers space, which becomes softer when water content increases. The anisotropic behaviour would result from interaction between matrix and joints deformation, which depends on loading orientation.

$\begin{array}{ll}\begin{array}{l}\text { Nomenclature } \\ \theta\end{array} & \begin{array}{l}\text { angle between the bedding plane and the } \\ \text { vertical axis }\end{array} \\ S & \text { suction (MPa) } \\ H & \text { relative humidity }(\% \mathrm{RH}) \\ w & \text { water content }(\%) \\ S & \text { degree of saturation } \\ \varepsilon & \text { strain } \\ L, T, T^{\prime} & \text { strain gauges } \\ \sigma & \text { stress } \\ E_{1}, E_{2} & \text { Young moduli } \\ G_{2} & \text { shear modulus } \\ v_{1}, v_{2} & \text { Poisson's ratios } \\ R_{\mathrm{tr}} & \text { triaxial compressive strength } \\ C & \text { cohesion } \\ \phi & \text { friction angle }\end{array}$

\section{Acknowledgements}

The authors wish to thank IRSN for providing financial assistance during this experimental program. They also thank Professor P. Bérest for his suggestions.

\section{References}

Al-Shayea, N., 2001. The combined effect of clay and moisture content on the behavior of remolded unsaturated soils. Engineering Geology 62, 319-342.

Bergues, J., Nguyen Minh, D., 1999. Comportement différé des argilites de l'Est: Influence du degré de saturation, écrouissage, extension, relaxation. Rapport LMS ANDRA no. D RP 0 LMS 99-001/A. (unpublished).

Cabrera Nunez, J., Beaucaire, C., Bruno, G., De Windt, L., Genty, A., Ramambasoa, N., Rejeb, A., Savoye, S., Volant, P., 2001. Projet Tournemire: synthèse des programmes de recherche 1995/1999. Rapport IPSN, 202 pp.

Charpentier, D., Tessier, D., Cathelineau, M., 2003. Shale microstructure evolution due to tunnel excavation after 100 years and 
impact of tectonic paleo-fracturing. Case of Tournemire, France. Engineering Geology 70, 55-69.

Cuisinier, O., Masrouri, F., 2003. Hydromechanical behaviour of a compacted swelling soil under very high suctions. Comptes Rendus de l'Academie des Sciences. Mecanique 331, 203-210.

Daupley, X., 1997. Etude du potentiel de l'eau interstitielle d'une roche argileuse et des relations entre ses propriétés hydriques et mécaniques. Thèse de l'Ecole Nationale Supérieure des Mines de Paris.

Delage, P., Howat, M.D., Cui, Y.J., 1998. The relationship between suction and swelling properties in a heavily compacted unsaturated soils. Engineering Geology 50, 31-48.

Niandou, H., Shao, J.F., Henry, J.P., Fourmaintraux, D., 1997. Laboratory investigation of the Mechanical Behaviour of Tourne- mire Shale. International Journal of Rock Mechanics and Mining Sciences 34, 3-16.

Ramambasoa, N., 2001. Etude du comportement hydromécanique des argilites: Application au site de Tournemire. Thèse de l'Ecole polytechnique.

Ramamurthy, T., 1993. Strength and modulus responses of anisotropic rocks. In: Hudson, J.A. (Ed.), Comprehensive Rock Engineering. Fundamentals, vol. 1. Pergamon, Oxford, pp. 313-329.

Rejeb, A., 1999. Mechanical characterisation of the argillaceous Tournemire site (France). In: Jha, P.C., Gupta, R.N. (Eds.). Proceedings of the International Conference on Rock Engineering Techniques for Site Characterisation, Bangalore/India/December 6-8, 1999. Oxford. 